\title{
Gap Influence on Wave Interaction of Twin Caissons in Close Proximity
}

\author{
Jingwen Zhang, Guanghua He, Lei Mei, and Dehe Zhang
}

\begin{abstract}
A two-dimensional incompressible viscous flow numerical wave tank by a CIP (Constraint Interpolation Profile) method is established which has capability of handling the violent free-surface flow problems. Wave resonance at the narrow gap between twin caissons in close proximity is simulated. Firstly, a fixed twin floating-body model is set up in the work area of the numerical wave tank. Systematic simulations of regular waves acting on the twin floating bodies with narrow gap are carried out with a wide range of incident wave frequencies. By changing the gap width and the draft of the twin floating caissons, separately, the mean wave heights in the gaps are found to agree reasonably well with the experimental results and the previous numerical simulations, which demonstrate that the CIP-based viscous model has the accuracy to predict the wave interaction of twin caissons in close proximity.
\end{abstract}

Index Terms-Resonance, gap influence, CIP, twin caissons, viscous.

\section{INTRODUCTION}

Gaps between the floating structures are widely existed in ocean engineering and coastal engineering. A water resonance at the gap may be induced by the incident waves in a proper situation. When the water resonance occurred in narrow gaps, wave height in the gap and the corresponding wave forces acting on the relative structures are dramatically increased. That may damage the structures. So it is significant to study the water resonance at the narrow gap and to investigate which physical parameters affect the resonance significantly.

Many studies have been done by many scholars and engineers [1]-[6]. Miao, Ishida, and Saitoh using the potential theory to study the wave force on the twin caissons with narrow gap between [1], [2]. Li, Cheng, and Deeks using a novel semi-analytical method named Scaled Boundary Element Method to study the gap resonance between two boxes [3]-[5]. Based on the same method, He,

Manuscript received November 21, 2016; revised May 21, 2017. This work was supported by National Natural Science Foundation of China (51579058), Shandong Provincial Natural Science Foundation (ZR2014EEQ016), Open Research Fund Program of Key Laboratory of Water \& Sediment Science and Water Hazard prevention (Changsha University of Science \& Technology), Hunan Province (2015SS02), Open Research Fund of State Key Laboratory of Satellite Ocean Environment Dynamics(Second Institute of Oceanography, SOA, SOED1514).

Jingwen Zhang, Lei Mei, and Dehe Zhang are with the School of Naval Architecture and Ocean Engineering, Harbin Institute of Technology, Weihai, China (e-mail: hitzhangjingwen@163.com; hit_marinehydro@163.com; hitparkzdh@163.com).

Guanghua $\mathrm{He}$ is with the School of Naval Architecture and Ocean Engineering, Harbin Institute of Technology, Weihai, China and State Key Laboratory of Satellite Ocean Environment Dynamics (Second Institute of Oceanography, SOA), Hangzhou, China (e-mail: ghhe@hitwh.edu.cn).
Teng, and Li calculated the hydrodynamics forces, reflection and transmission coefficients when the gap resonant in gap of twin floating boxes [6]. Zhu, and Miao based on the 3D frequency domain method to investigate the effect of the size of narrow gap on the hydrodynamic forces on the multi-floating-bodies [7]. Ning, Su, and Teng using a fully nonlinear potential theory to simulate water resonance in narrow gap [8], [9].

The researches mentioned above are all based on the potential theory, which has an advantage of CPU time-saving but has the biggest disadvantage of ignoring the viscous of the fluid. It is reported that the computational results of wave height at the gap by the potential theory at the resonant frequency are over-predicted 10 times [1], [2]. Then, many scholars began to use the damping coefficient and viscous flow to investigate the gap resonance from the practical view of point. Lu, Teng, and Cheng based on the viscous flow model to study the gaps in three floating bodies [10], [11]. Chen, Chen, and Zhan used the commercial software FLUENT to simulate the hydrodynamics performance of two floating bodies in proximity [12]. Hu, Kashiwagi, and Kitadai using a CIP (Constraint Interpolation Profile) to simulate a violent free surface flow problem [13]. It shows the powerful performance of the CIP method in simulate the strongly nonlinear problem. So, the CIP is also introduced in the present study. Firstly, a viscous-flow-based numerical wave tank is constructed. Then, intensive computations are carried with different gap width and draft of caissons. The wave height at the narrow gap between two caissons are illustrated and compared with available published experimental data [14] and numerical results [10], [11]. Satisfactory agreement shows that the CIP-based viscous numerical wave tank has the capability of handing water resonance in narrow gap between twin caissons with viscous effect considered.

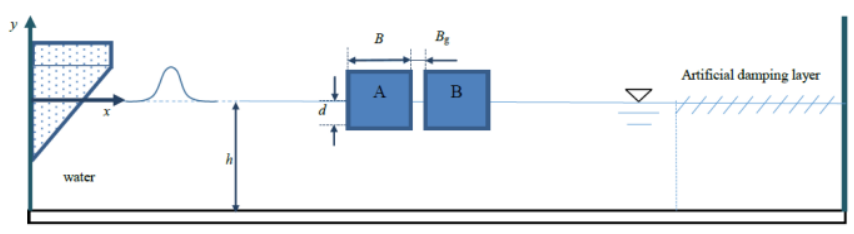

Fig. 1. Schema of 2D numerical wave tank.

\section{NUMERICAL WAVE TANK}

\section{A. Numerical Model Setup}

As shown in Fig. 1, numerical simulations are carried out in a two-dimensional numerical wave tank. Waves are produced by a plunger type wave generator located at the left end of the wave tank. Artificial damping layer which is $6.6 \mathrm{~m}$ is configured at the right of the tank for the sake of wave 
absorbing. A right-hand Cartesian coordinate system oxy is defined on the undisturbed free surface. The $o x$ axis is along the free surface which positively point to the right and oy axis is then on the left wall of the wave tank pointing upward.

\section{B. Governing Equations}

Assuming that the fluid is incompressible, the governing equations given by continuity equation and Navier-Stokes equations can be expressed as follows:

$$
\frac{\partial u_{i}}{\partial x_{i}}=0
$$

(a)

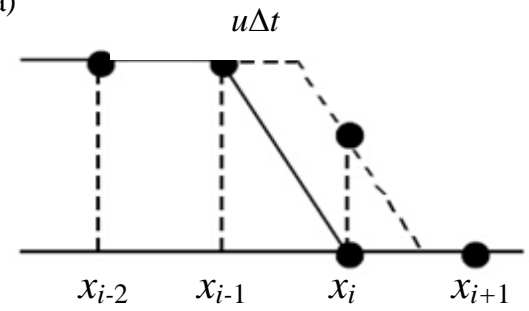

(c)

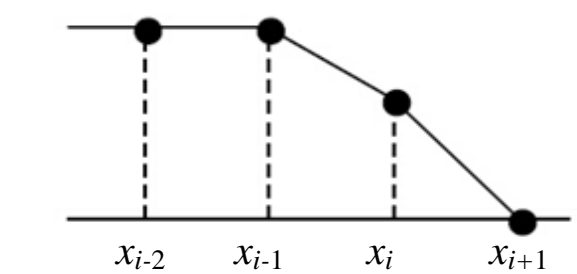

3) Non-advection Step (ii)

$$
\frac{u_{i}^{n+1}-u_{i}^{* *}}{\Delta t}=-\frac{1}{\rho} \frac{\partial p^{n+1}}{\partial x_{i}}
$$

In the advection step, the calculation is implemented by the CIP scheme. The pressure-velocity coupling is treated in a non-advection step calculation, in which the following Poisson equation is solved.

$$
\frac{\partial}{\partial x_{i}}\left(\frac{1}{\rho} \frac{\partial p^{n+1}}{\partial x_{i}}\right)=\frac{1}{\Delta t} \frac{\partial u_{i}^{* *}}{\partial x_{i}}
$$

(b)

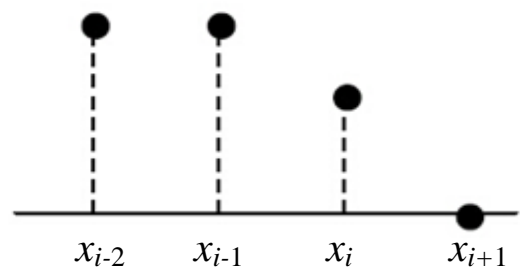

(d)

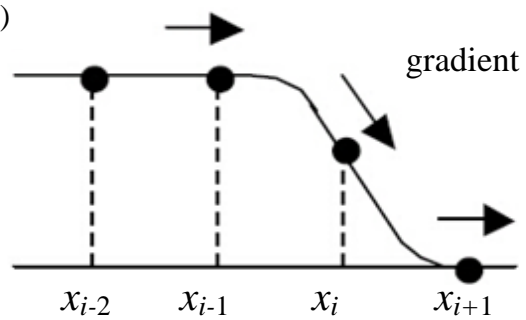

Fig. 2. The principle of the 1D CIP method.

(a) The solid line is the initial profile and the dashed line is an exact solution, (b) profile at discretized points after advection, (c) profile by linearly interpolated, (d) In the CIP, the spatial derivative also propagates and the profile inside a grid cell is retrieved.

$$
\frac{\partial u_{i}}{\partial t}+u_{j} \frac{\partial u_{i}}{\partial x_{j}}=-\frac{1}{\rho} \frac{\partial p}{\partial x_{i}}+\frac{1}{\rho} \frac{\partial}{\partial x_{j}}\left(\mu S_{i j}\right)+f_{i}
$$

where, $\rho$ is the density of fluid; $u_{i}(i=1,2,3)$ is the velocity component; $S_{i j}=\partial u_{i} / \partial x_{j}+\partial u_{j} / \partial x_{i}$; and $f_{i}$ in Eq. (2) denotes the body force, such as the gravity force etc. Time evaluation of Eq. (2) is performed by a fractional step method. The calculation of equation is divided into an advection step and two non-advection steps, expressed as follows,

1) Advection Step

$$
\frac{u_{i}^{*}-u_{i}^{n}}{\Delta t}+u_{j}^{n} \frac{\partial u_{i}^{n}}{\partial x_{j}}=0
$$

2) Non-advection Step (i)

$$
\frac{u_{i}^{* *}-u_{i}^{*}}{\Delta t}=\frac{1}{\rho} \frac{\partial}{\partial x_{j}}\left(\mu S_{i j}^{*}\right)+f_{i}
$$

Eq. (6) is assumed to be valid for liquid, gas and solid phases. Solution of it gives the pressure distribution in the whole computation domain.

\section{Initial and Boundary Condition}

The numerical computation starts from the static state, which means the initial velocities of the fluid and caissons are zero. The wave generator starts gradually from zero to the expected amplitude of motion in order to ensure numerical stability of the simulation. It is supposed that there are neither inflows nor outflows in the inlet and outlet of the wave tank and the non-slip boundary condition is imposed on the caissons' surface.

\section{CIP Method}

In order to understand the CIP method, 1D advection equation is briefly introduced, and more detailed information can be referred to Takiwaki [15] and $\mathrm{Hu}$ [16],

$$
\frac{\partial f}{\partial t}+u \frac{\partial f}{\partial x}=0
$$




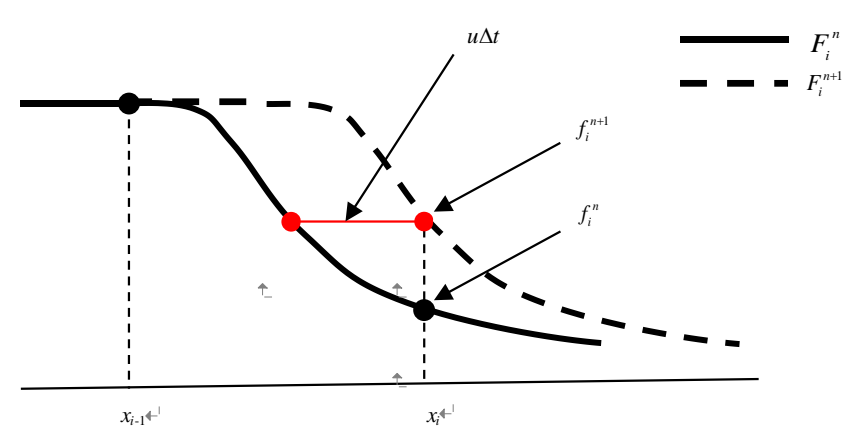

Fig. 3. CIP scheme as a kind of Semi-Lagrangian method.

As shown in Fig. 2 (a)-(c), a first-order upwind scheme, which can be constructed by adjacent two grid points, results in numerical diffusion. We find a smoother profile of $f$ after time stepping. The CIP scheme, which is illustrated in Fig. 2 (d), shows a different way by using the grid point value and its spatial derivative in two grid points to form a cubic polynomial to approximate the profile. By differentiating Eq. (7) with respective to $x$, we have the equation about the spatial derivative as:

$$
\frac{\partial g}{\partial t}+u \frac{\partial g}{\partial x}=-g \frac{\partial u}{\partial x}
$$

where $g=\partial f / \partial x$. For the case of $u>0$, a profile for $f^{\text {n }}$ inside the upwind cell $\left(x_{i-1}, x_{i}\right)$ may be approximated as:

$$
F_{i}^{n}(x)=a_{i}\left(x-x_{i}\right)^{3}+b_{i}\left(x-x_{i}\right)^{2}+c_{i}\left(x-x_{i}\right)+d_{i}
$$

As shown in Fig. 3 the profile at the time step $n+1$ is obtained by shifting the profile with $-u \Delta t$. The time evolution of the function $f$ and $g$ can be obtained by using the following Lagrangian invariants.

$$
\begin{gathered}
f^{n+1}(x)=F^{n}(x-u \Delta t) \\
g^{n+1}(x)=g_{i}^{n}(x-u \Delta t)
\end{gathered}
$$

The four unknown coefficients in Eq. (9) can be determined by using known quantities $f_{i}^{n}, f_{i-1}^{n}, g_{i}^{n}$, and $g_{i-1}{ }^{\mathrm{n}}$.
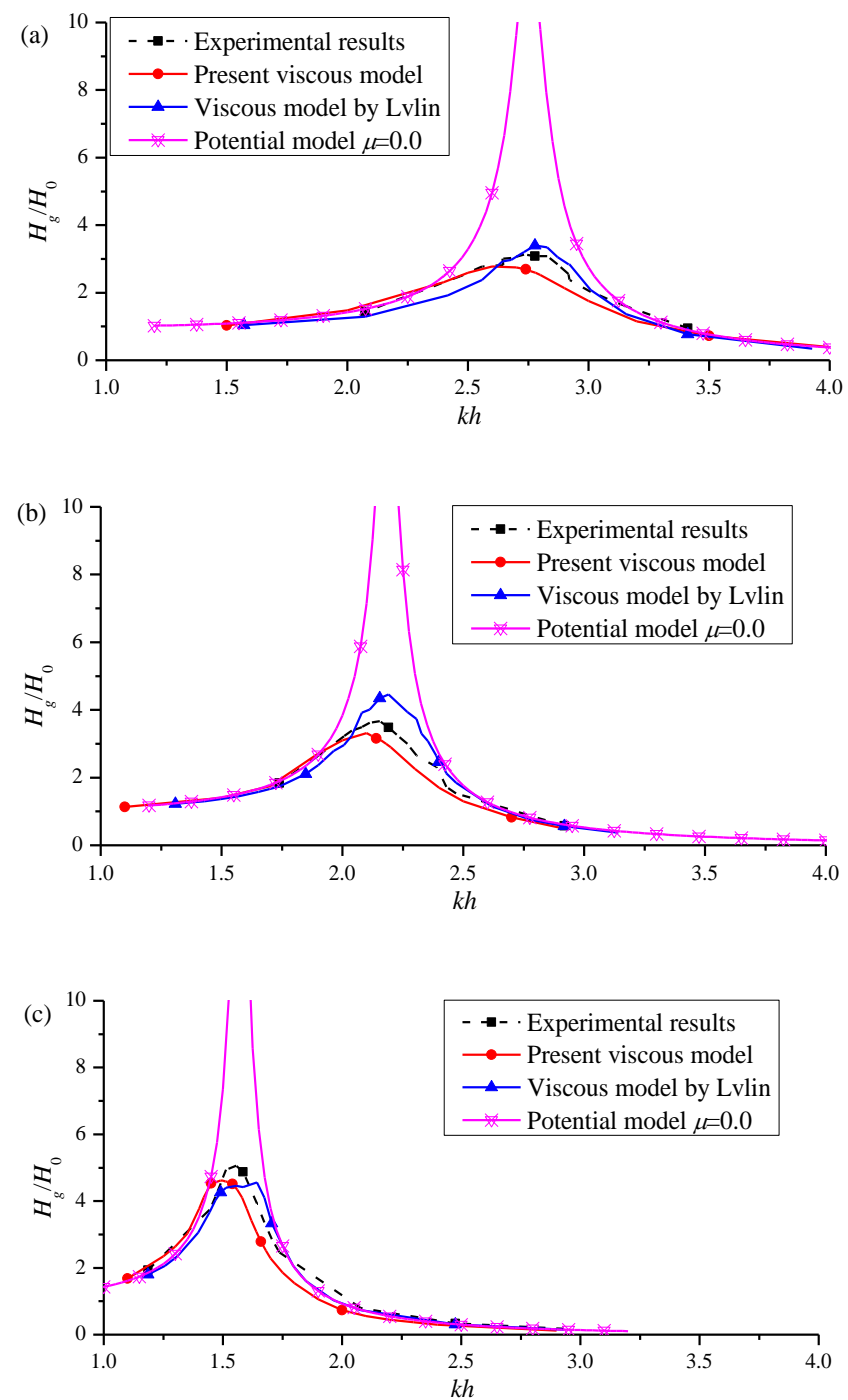

\begin{tabular}{|c|c|c|c|c|c|c|c|}
\hline & $B_{\mathrm{g}}(\mathrm{m})$ & 0.03 & relative error(\%) & 0.05 & relative error(\%) & 0.07 & relative error $(\%)$ \\
\hline \multirow[t]{3}{*}{$k h$} & Experimental result ${ }^{[14]}$ & 1.639 & & 1.566 & & 1.469 & \\
\hline & Present viscous model & 1.615 & -1.46 & 1.500 & -3.60 & 1.420 & -3.33 \\
\hline & Viscous model by Lvlin ${ }^{[10]}$ & 1.735 & 5.85 & 1.643 & 5.59 & 1.494 & 1.70 \\
\hline \multirow[t]{3}{*}{$H_{\mathrm{g}} / H_{0}$} & Experimental result $^{[14]}$ & 4.512 & & 5.060 & & 4.672 & \\
\hline & Present viscous model & 4.506 & -0.13 & 4.618 & -8.73 & 4.705 & 0.70 \\
\hline & Viscous model by Lvlin ${ }^{[10]}$ & 4.790 & 6.16 & 4.556 & -9.90 & 4.450 & -4.75 \\
\hline
\end{tabular}

Fig. 4. The mean wave height in the narrow gap with incident wave frequency.

TABLE I: The DimensionLess Resonant FREQUENCIES AND WAVE HeIGHTS

\section{NUMERICAL RESULTS AND DISCUSSIONS}

As shown in Fig. 1, the length of the numerical wave tank is set as $17.38 \mathrm{~m}$ long with water depth $h=0.5 \mathrm{~m}$. Twin caissons with the same breadth $B=0.5 \mathrm{~m}$ are fixed in the numerical wave tank, which are in accordance with the parameter sets of Saitoh [14] and Lvlin [10] for the purpose of comparison. A non-uniform grid is employed for the computational domain with $587 \times 284$ in the $x$ - and $y$-axes, respectively. The grid is concentrated with minimum spacing $x=0.001 \mathrm{~m}$ and $y=0.0005 \mathrm{~m}$. The time step is set as $1 / 2000$. Different gap width $B_{\mathrm{g}}$ and drafts $d$ are considered in the present numerical model to investigate the influences on wave interaction

\section{A. Influence of Gap Width}

The influence of gap width on the surface elevation at the gap between two caissons is investigated. Three different gap width $B_{\mathrm{g}}=0.03,0.05,0.07 \mathrm{~m}$ are considered with the fixed 
draft of the caissons $d=0.252 \mathrm{~m}$. Fig. 4 show the variations of non-dimensional mean wave height $H_{\mathrm{g}} / H_{0}$ in the narrow gap against the non-dimensional wave number $k h$. Four curves in Fig. 4 represent the experimental results by [14], the present viscous flow numerical results, the viscous flow results by Lvlin [10], and potential flow results, respectively.

It appears that both the viscous flow model and the potential flow model can predict the resonant frequency in the narrow gap with accuracy. The potential flow model over-predicts the wave height in narrow gap at the frequencies close to the resonant frequency as a consequence of regardless of the physical energy dissipation, vortex shedding, and turbulence. To further analyze the present viscous model, a comparative study of the resonant frequencies and resonant wave heights obtained from Fig. 4 are summarized in Table I, which also includes the relative error between the numerical results and the experimental results. The relative error is defined as follow,

relative error $=\frac{\text { numerical result }- \text { experimental result }}{\text { experimental result }} \times 100$

The comparison shown in Fig. 4 and Table I indicate that both the present viscous flow model and the viscous flow model by Lvlin [10] perform well in simulating the fluid resonance in narrow gaps. The curve of the present viscous model smoother than the viscous model by Lvlin [10], and there is a certain phase shift between them. Most of the time, the relative error of the present viscous model is far below that of the viscous model by Lvlin [10], and the relative error of wave height can even as low as $-0.13 \%$ when $B_{\mathrm{g}}=0.03 \mathrm{~m}$, which indicate that the present viscous model have greater accuracy in predicting the resonant frequency and corresponding wave height in the narrow gap.
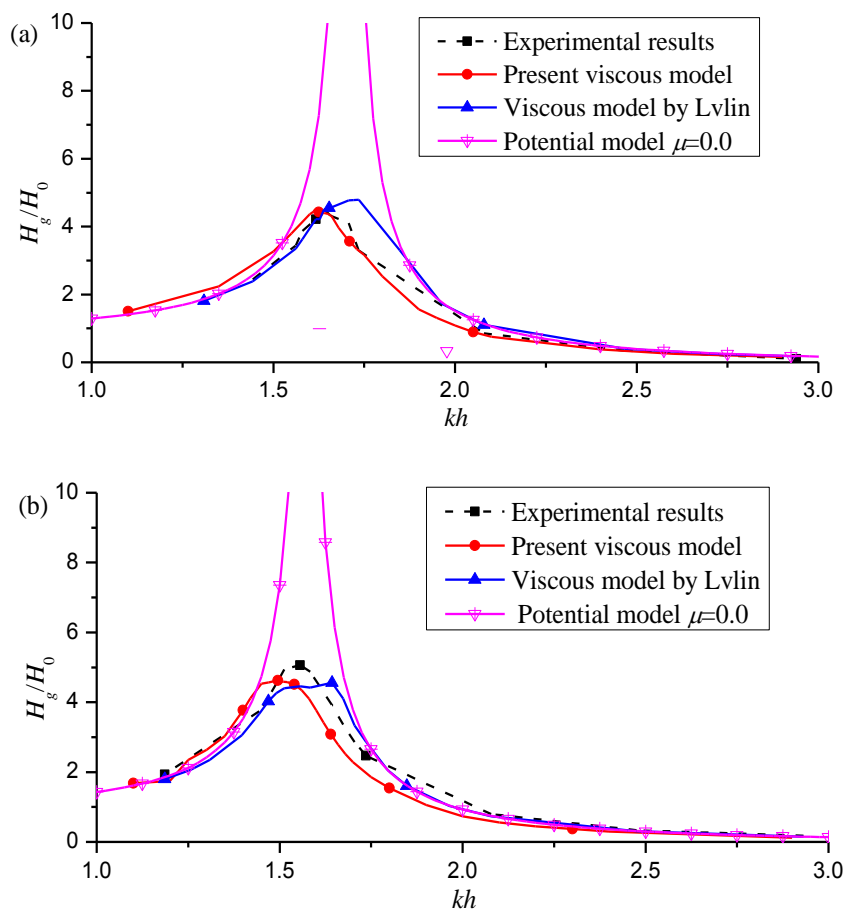

Fig. 5. The mean wave height in the narrow gap with incident wave frequency.

TABLE II: THE DiMENSIONLESS RESONANT FREQUENCIES AND WAVE HEIGHTS

\begin{tabular}{|c|c|c|c|c|c|c|c|}
\hline & $d(\mathrm{~m})$ & 0.103 & relative error $(\%)$ & 0.153 & relative error $(\%)$ & 0.252 & relative error(\%) \\
\hline \multirow[t]{3}{*}{$k h$} & Experimental result ${ }^{[14]}$ & 2.732 & & 2.154 & & 1.469 & \\
\hline & Present viscous model & 2.600 & -4.83 & 2.100 & -2.506 & 1.420 & 2.110 \\
\hline & Viscous model by Lvlin ${ }^{[10]}$ & 2.788 & 1.68 & 2.191 & 1.717 & 1.494 & 1.701 \\
\hline \multirow[t]{3}{*}{$\overline{H_{\mathrm{g}} / \mathrm{H}_{0}}$} & Experimental result $^{[14]}$ & 3.130 & & 3.688 & & 4.672 & \\
\hline & Present viscous model & 2.766 & -11.13 & 3.312 & -9.705 & 4.705 & -1.155 \\
\hline & Viscous model by Lvlin ${ }^{[10]}$ & 3.396 & 8.49 & 4.457 & 21.510 & 4.450 & -4.751 \\
\hline
\end{tabular}

\section{B. Influence of Caisson Draft}

As shown in Fig. 5, in order to investigate the influence of caisson draft on the water resonance at the gap, we examine three different drafts, i.e., $d=0.103,0.153$, and $0.252 \mathrm{~m}$, while the gap width is set to be a constant of $B_{\mathrm{g}}=0.05 \mathrm{~m}$. As before, the four curves in the graph represent the experimental results, the present viscous flow numerical results, the viscous flow results by Lvlin [10], and potential flow results, respectively.

Similar to the previous section, both the potential flow result and the viscous flow result are in general accordance to the experimental data [14] at either side of the resonant frequency. The potential model succeeds in predicting the resonate frequency like viscous model but fails to predict the resonant wave heights reasonably. With the increase of draft, the resonant frequency band narrows down and the resonant frequency becomes lower obviously. The curve of the present viscous model smoother than the viscous model by Lvlin [10] and there is a certain phase shift between them. According to the above study it confirms that the present viscous model has relatively accurate prediction in simulating the fluid resonance in narrow gap.

\section{CONCLUSION}

Numerical simulations based on the viscous flow theory aimed at investigating the wave height at the gap between twin caissons were conducted in the wave tank by changing gap width and caissons draft. Owing to the application of CIP method, high precision was obtained in predicting wave frequencies and relevant wave heights in narrow gaps. The maximum relative error of the former was $-4.83 \%$, while the latter was $11.13 \%$. With the increase of gap width and caisson draft, the resonant frequency becomes lower and the frequency band causing resonance narrows down. In 
conclusion, this research work can provide somewhat guidance for the engineering application.

\section{ACKNOWLEDGMENT}

This work was supported by National Natural Science Foundation of China (51579058), Shandong Provincial Natural Science Foundation (ZR2014EEQ016), Open Research Fund Program of Key Laboratory of Water \& Sediment Science and Water Hazard prevention (Changsha University of Science \& Technology), Hunan Province (2015SS02), Open Research Fund of State Key Laboratory of Satellite Ocean Environment Dynamics (Second Institute of Oceanography, SOA, SOED1514).

\section{REFERENCES}

[1] G. Miao, H. Ishida, and T. Saitoh, "Influence of gaps between multiple floating bodies on wave forces," China Ocean Engineering, vol. 14, no. 4, pp. 407-422, 2000.

[2] G. Miao, T. Saitoh, and H. Ishida, "Water wave interaction of twin large scale caissons with a small gap between," Coastal Engineering Journal, vol. 43, no. 1, pp. 39-58, 2001.

[3] B. Li, L. Cheng, and A. J. Deeks, "A modified scaled boundary finite-element method for problems with parallel side-faces-Part I: Theoretical developments," Applied Ocean Research, vol. 27, no. 4, pp. 216-223, Aug. 2005.

[4] B. Li, L. Cheng, and A. J. Deeks, "A modified scaled boundary finite-element method for problems with parallel side-faces-Part II Application and evaluation," Applied Ocean Research, vol. 27, no. 4, pp. 224-234, Aug. 2005.

[5] B. Li, L. Cheng, and A. J. Deeks, "A semi-analytical solution method for two-dimensional Helmholtz equation," Applied Ocean Research, vol. 28, no. 3, pp. 193-207, June 2006.

[6] G. H. He, B. Teng, and B. N. Li, "Research on the hydrodynamic influence from the gaps between three identical boxes by a scaled boundary finite element method," Journal of Hydrodynamics, vol. 21, no. 3, pp. 418-424, May 2006. (In Chinese)

[7] R. C. Zhu, H. R. Zhu, and G. Miao, "Influences on hydrodynamics of multiple floating structures with small gap in between," Journal of Shanghai Jiaotong University, vol. 42, no. 8, pp. 1238-1242, Aug. 2008. (in Chinese)

[8] D. Z. Ning, X. J. Su, and B. Teng, "Numerical study of fluid resonance induced by wave action on multi-boxes with narrow gaps," Haiyang Xuebao, vol. 37, no. 3, pp. 126-133, Mar. 2015.

[9] X. J. Su, D. Z. Ning, and B. Teng, "Analysis of waves loads on multi-boxes with narrow gaps," Engineering mechanics, vol. 33, no. 4, pp. 233-240, Apr. 2016.

[10] L. Lu, B. Teng, and L. Cheng, "Modelling of multi-bodies in close proximity under water waves-Fluid resonance in narrow gaps," Science China, vol. 54, no. 1, pp. 16-25, Jan. 2011.

[11] L. Lu, B. Teng, and L. Sun, "Modelling of multi-bodies in close proximity under water waves - Fluid forces on floating bodies," Ocean Engineering, vol. 38, no. 13, pp. 1403-1416, July. 2011.

[12] X. B. Chen, Z. Y. Chen, and J. M. Zhan, "Numerical investigation of the resonance phenomenon between waves and floating bodies," Chinese Journal of Hydrodynamics, vol. 29, no. 3, pp. 338-345, May. 2014. (in Chinese)

[13] C. Hu, M. Kashiwagi, and A. Kitadai, "Numerical simulation of strongly nonlinear wave-body interactions with experimental valilotion," in Proc. the 3rd Asia-Pacific Workshop on Marine Hydrodynamics, 2006, pp. 232-236.
[14] T. Saitoh, G. P. Miao, and H. Ishida, "Theoretical analysis on appearance condition of fluid resonance in a narrow gap between two modules of very large floating structure," in Proc. the $3 r d$ Asia-Pacific Workshop on Marine Hydrodynamics, 2006, pp. 170-175.

[15] H. Takewaki, A. Nishiguti, and T. Yabe, "Cubic interpolated pseudo-particle method (CIP) for solving hyperbolic-type equations," Journal of Computational Physics, vol. 61, no. 2, pp. 261-268, Mar. 1985.

[16] C. H. Hu and M. Kashiwagi, "A CIP-based method for numerical simulations of violent free-surface flows," Journal of Marine Science and Technology, vol. 9, no. 4, pp. 143-157, Mar. 2004.

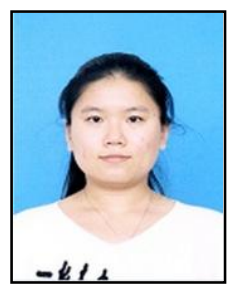

Jingwen Zhang was born on June 9, 1995, in Shandong, China. She is an undergraduate student at Harbin Institute of Technology, Weihai currently. She focuses on the research of architecture and ocean engineering.

She doesn't have a job yet. She would like to devote herself into hydrodynamics.

Ms. Zhang doesn't have any memberships in professional societies.

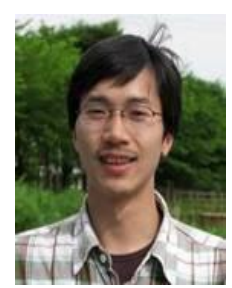

Guanghua He was born in Zhejiang province, China, 1980. His educational background is as follows: 1999.09-2003.07 Tianjin University, Bachelor of port, coastal and offshore engineering.

He received his master of port, coastal and offshore engineering from Dalian University of Technology from 2003 to 2006; He received his doctor degree in marine atmospheric environment engineering from Dalian University of Technology from 2006 to 2009.

He started his career in 2009. His work experience is as follows: 2009.11-2014.03 Osaka university, assistant professor of ship and ocean engineering. From 2014.05 until now, School of Naval Architecture and Ocean Engineering, Harbin institute of technology, weihai, professor, doctoral tutor. His research interests are (1) Strongly nonlinear ship motion and hydrodynamic problems, (2) The ship's seakeeping and wave load, (3) The fluid-solid coupling water elastic analysis.

Prof. He is the member of International Society of Ocean and Polar Engineering.

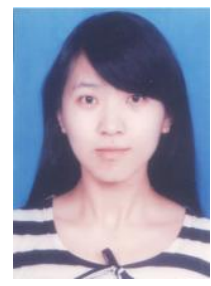

Lei Mei was born in Shandong province, China, 1981 Her received her bachelor of composite material, master of materials science, doctor degree in materials science from Harbin Institute of Technology, in 2004 2007, 2010.

From 2010.10 until now, School of Naval Architecture and Ocean Engineering, Harbin institute of technology, weihai, lectureship. Her research interest are (1) Modeling processing of composite material propeller, (2) Design resrarch of high-speed ship in composite material.

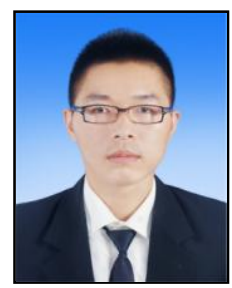

Dehe Zhang was born in Shandong province, China, 1991. He is a postgraduate student at Harbin Institute of Technology, Weihai currently. $\mathrm{He}$ focuses on the research of architecture and ocean engineering. 